\title{
Change in Material Properties at Mechanoactivation
}

ISSN: 2576-8840

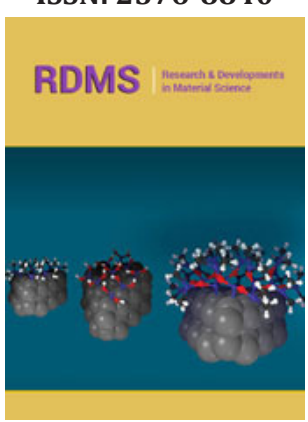

*Corresponding author: Pryadko Nataliya Sergeevna, Institute of Technical Mechanics of National Academy of Science of Ukraine and State space Agency of Ukraine, Ukraine

Submission: 海June 02, 2021

Published: 制June 10, 2021

Volume 15 - Issue 3

How to cite this article: Pryadko Nataliya Sergeevna. Change in Material Properties at Mechanoactivation. Res Dev Material Sci. 15(3). RDMS.000862. 2021. DOI: 10.31031/RDMS.2021.15.000862

Copyright@ Pryadko Nataliya Sergeevna. This article is distributed under the terms of the Creative Commons Attribution 4.0 International License, which permits unrestricted use and redistribution provided that the original author and source are credited.

\section{Pryadko Nataliya Sergeevna*}

Institute of Technical Mechanics of National Academy of Science of Ukraine and State space Agency of Ukraine, Dnipro, Ukraine

\begin{abstract}
Grinding activation is a new way to accelerate physical and chemical processes. It finds more and more widespread use and is the basis of fundamentally new technological processes, when an auxiliary operation becomes the main one. In the production of cement, crushed granular blast furnace slag can partially replace cement, provided the desired specific surface area of the product. This operation reduces energy consumption for the technological process and leads to lower consumption of natural raw materials.

The studies of the dependence of the activated material properties on its storage terms have been carried out. Analysis of the results allows us to assert that the specific surface area of blast-furnace slags for each type of material decreases during the storage of crushed powders. The established dependencies can be used to predict the permissible storage and storage time of activated finely dispersed materials for their further use as building materials and mineral powders for construction and production of building materials.
\end{abstract}

Keywords: Activation; Grinding; Material properties; Blast-furnace slag

\section{Introduction}

Mechanical activation is an activation of solids by mechanical processing. Milling in shock, shock-abrasive or abrasive modes leads to the accumulation of structural defects, an increase in surface curvature, phase transformations and even crystal amorphization, which affects their chemical activity. Therefore we can say that mechanical activation is the process of formation of a substance with a higher chemical activity due to preliminary mechanical processing [1].

The concept of "mechanical activation" was introduced into the literature by A. Smekal [2]. This process is defined as a change in the energy state, physical structure and chemical properties of mineral substances under the action of mechanical forces during dispersion, and the change in the energy state refers to a heterophase system, the solid components of which were subjected to mechanical action. Introduction to the mechanical activation definition of the energy system state opens up the possibility of mathematical expression and quantitative assessment of activation: mechanical activation is numerically equal to the change in the free energy of the system under the action of mechanical forces.

Part of the mechanical energy supplied to a solid during activation is assimilated by it in the form of a new surface, linear and point defects. The consequences of mechanical chemical activation [3] are manifested in the improvement of the mechanical properties of materials (decrease in porosity, increase in strength and improvement of plastic properties, etc.), increase in the reactivity of solid reagents, decrease in the sintering temperature of grinding products, etc.

One of the main mechanical activation theses is that mechanical activation can be without grinding, but grinding cannot be without activation. Hence, it follows that, firstly, grinding 
and activation cannot be divided: any grinding is activation, since under the action of external forces the energy reserve of the ground substance increases, at least due to an increase in surface energy; secondly, any grinding apparatus is a mechanical activator. Grinding in any apparatus activates the processed material to a greater or lesser extent.

Mechanical activation occurs when the accumulation rate of defects exceeds the rate of their disappearance. This is realized in power-loaded devices: centrifugal, planetary and jet mills, disintegrators, which combine high frequency and force of mechanical action [2].

Activation by grinding as a new way to accelerate physicochemical processes is finding more and more application. It has already gone beyond the framework of laboratory research and is used as a means of accelerating technological processes or as a way to change the technological parameters of the processing mode of mineral raw materials.

Activation by grinding (crushing) finds application in solving problems of the complex use of mineral resources and reducing the harmful effects of industrial processing products on the environment. In particular, it can be the utilization of production waste and the elimination of dumps; wastewater treatment with the capture of valuable (and harmful) components on the activated surface; upgrading of peat, coal and oil shale before combustion with simultaneous extraction of metals, sulfur and other valuable components; replacing the roasting of sulfide and arseniccontaining concentrates with a non-roasting process based on mechanical activation [4].

Utilization of blast-furnace slag accumulating in dumps allows solving environmental problems of industrial regions of Ukraine and Europe at all. At the same time it expands the raw material base for the production of building materials - slag-alkaline binders. According to the conditions of hardening and water resistance of the product, these materials are hydraulically active and are obtained by mixing ground granular slag with alkaline metal compounds, or joint grinding of the slag with low-hygroscopic alkaline components [5]. The raw material base for the production of slag-alkaline concretes is practically unlimited due to the use of slag from various technological processes of black and nonferrous metallurgy: blast-furnace, electrothermophosphoric, open-hearth, converter, aluminothermal production, etc.

The purpose of this work is to study the possibility of mechanoactivation of blast-furnace slag of different storage periods.

\section{Experimental Materials}

Blast furnace slag is a by-product of the blast furnace cast iron production process. Raw charge for iron smelting consists of iron ore, coke and flux (limestone, dolomite and other materials). The air-cooled slag cools slowly and acquires a crystalline structure. After crushing, this slag can be used as building materials. Fireliquid blast-furnace slag is granulated in a short time according to the scheme of wet or semi-dry granulation and solidifies in the form of glassy granules. Due to this mineralogical structure, granular slags exhibit the highest latent hydraulic properties, which allow them to be used as an effective hydraulic additive in cement production [6]. In [3,7], an improvement in the binding properties of crushed building materials was established: technical alumina, mullite-corundum chamotte, cement, slag, molding gypsum. In the range of the specific surface area of crushed cement $\mathrm{S}_{\mathrm{sp}}=0.4$ $0.7 \mathrm{~m}^{2} / \mathrm{g}$, there is a decrease in flowability, an increase in plastic viscosity and reduction in the cementation in 1.5-2 times.

Several factors are greatly influenced on the physical state and chemical properties of the material upon activation, among which the most important are the dispersion of the ground substance and the shelf life. Experimental studies were carried out on the activation of slag of the Metallurgical Plant (city of Mariupol). Blast furnace and granular slag of different initial size and shelf life were considered. Table 1 shows the granulometric compositions of the four investigated materials.

Table 1: Size composition of metallurgical slag.

\begin{tabular}{|c|c|c|c|c|}
\hline Size Class, $\mathbf{m m}$ & Blast Furnace Slag, $\boldsymbol{\gamma}, \boldsymbol{\%}$ & Old Blast Furnace Slag, $\boldsymbol{\gamma}, \boldsymbol{\%}$ & Granulated Slag $\boldsymbol{\gamma}, \boldsymbol{\%}$ & Old Granulated Slag, $\boldsymbol{\gamma}, \mathbf{3}$ \\
\hline & $\mathbf{1}$ & $\mathbf{2}$ & $\mathbf{3}$ & $\mathbf{4}$ \\
\hline$+10,0$ & 0,5 & 24,1 & 4,0 & 6,7 \\
\hline$-10,0+5,0$ & 4,2 & 17,5 & 3,9 & 7,0 \\
\hline$-5,0+3,0$ & 7,5 & 9,9 & 14,7 & 31,1 \\
\hline$-3,0+1,0$ & 32,9 & 15,1 & 26,6 & 25,3 \\
\hline$-1,0+0,45$ & 33,7 & 12,3 & 16,8 & 11,8 \\
\hline$-0,45+0,25$ & 15,4 & 9,9 & 8,9 & 3,1 \\
\hline$-0,25+0,15$ & 3,4 & 5,9 & 8,7 & 1,4 \\
\hline$-0,15$ & 2,4 & 5,3 & \\
\hline
\end{tabular}

With an increase in the content of blast-furnace (and granulated) slag in slag cements, the properties of concrete are characterized by general operational data:
1. the longer cementation ensures the mobility of the mixture for a longer period, that is a special advantage in the summer, and with prolonged hardening, the strength increases - a consequence of the hydration of the slag; 
2. the hydration heat decreases, therefore, slag cements with a high slag content are preferably used in the production of mass concrete structures, which reduces the risk of thermal destruction;

3. the strength decreases in the initial period of hardening, however, 28-day strength remains unchanged at the same standard strength of cements;

4. the physical and mechanical properties of concrete, such as elasticity, creep and shrinkage, do not change significantly.

\section{Research Results}

An important problem is the change in the properties of activated slag during long-term storage (shelf life). Therefore, studies of the properties of crushed slag of four different types were carried out for a month. Since the level of mechanical activation can be determined by the value of the newly formed specific surface during grinding, then the specific surface area of the activated powders was measured at the control time. The dependences of the change in the specific surface area of blast-furnace slag on the shelf life are shown in Figure $1 \&$ in Table 2.

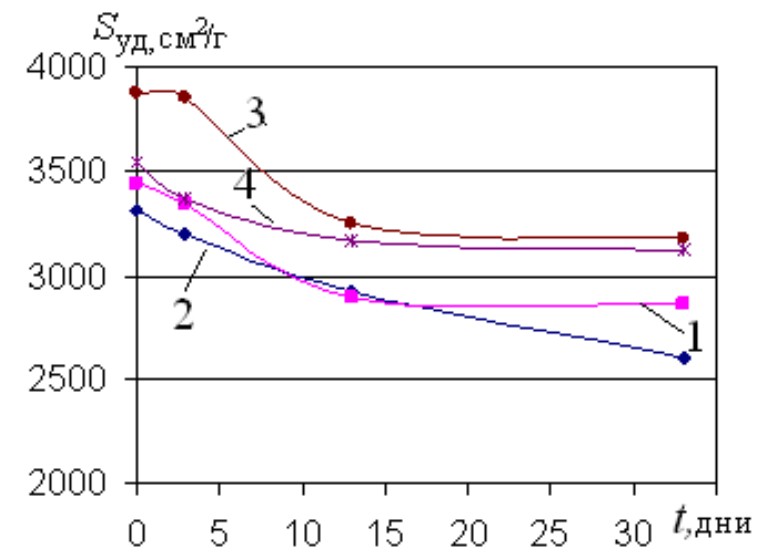

Figure 1: Kinetics of changes in the specific surface area of blast furnace slag samples.

Table 2: Change in the specific surface of slag over time.

\begin{tabular}{|c|c|c|c|c|}
\hline \multirow{2}{*}{$\begin{array}{c}\text { Sample } \\
\text { Number }\end{array}$} & \multicolumn{4}{|c|}{ Shelf Life, Days } \\
\cline { 2 - 5 } & $\mathbf{0}$ & $\mathbf{3}$ & $\mathbf{1 3}$ & $\mathbf{3 3}$ \\
\cline { 2 - 5 } Specific surface of samples, $\mathbf{c m}^{2} / \mathbf{g}$ \\
\hline 1 & 3441 & 3347 & 2886 & 2852 \\
\hline 2 & 3310 & 3199 & 2920 & 2601 \\
\hline 3 & 3872 & 3854 & 3248 & 3177 \\
\hline 4 & 3546 & 3369 & 3168 & 3123 \\
\hline
\end{tabular}

Analysis of the results showed that the dependences have a quadratic form $S_{s p}=a t^{2}-b t+c$, where the coefficients depend on the type of starting material and its size. So, for blast-furnace slag this is the equation $S_{s p}=0.43 t^{2}-35.4 t+3306$, and for granular slag $S_{s p}=0.8 t^{2}-38.4 t+3518$ with an approximation accuracy of $\mathrm{R}=0.99$.

The properties of activated materials depend on the initial properties of blast-furnace slag; stale (old) raw materials are less susceptible to mechanical activation. However, by choosing the grinding mode, the desired level of activation can be achieved. For example, samples 1 and 2, as well as 3 and 4, respectively, have similar specific surface area values (Table 2). With further storage, the dependence nature of the product specific surface kinetics on the shelf life does not differ significantly.

\section{Conclusion}

Activation by grinding may be the basis of fundamentally new technological processes, when an auxiliary operation becomes the main one. In the production of cement, crushed granular blast furnace slag can partially replace cement, provided the required specific surface area of the obtained product, that reduces the consumption of energy resources for the technological process and leads to lower costs of natural raw materials.

The analysis of the study results allows to state that the specific surface area of blast-furnace slag for each type of material decreases during the storage of crushed powders. The established dependencies can be used to predict the permissible storage and shelf life of activated fine-dispersed materials for their further use as binding materials and mineral powders for construction and production of building materials.

\section{References}

1. Lyakishev NP (2000) Encyclopedic Dictionary of Metallurgy. Intermet Engineering.

2. Bilenko LF (2009) Mechanical activation at dispersing solid materials.

3. Pilov PI, Gorobets LZh, Gayevoy VV, Pryadko NS, Shulyak IA (2007) Mechanical activation during grinding of minerals. Bulletin of KTU 16: 59-65.

4. Fine grinding and certification of powders.

5. Hobotova EB, Kalmykova YuS, Belichenko EA, Baumer VN (2013) Waste blast-furnace slags as technogenic raw materials for the production of slag-alkaline binders. Energy Technologies and Resource Saving 2: 4753.

6. Härdtl R (2003) Slag cements: general provisions. Cement and its application 1: 15-17.

7. Gorobets LZh, Kovalenko VV, Pryadko NS, Kravchenko VP (2009) Strengthening of building materials during treatment in jets. $\mathrm{Zb}$ naukovykh prats PolNTU 3 (25): 59-66. 